\title{
DEL BIPARTIDISMO A LA PLURALIDAD POLÍTICA EN LA COMUNIDAD DE MADRID: CAMBIOS TERRITORIALES (2011-2019)
}

\author{
Julio Fernández Portela \\ Grupo de Investigación Geografía, Paisaje y Tecnologías \\ de la Información del Departamento de Geografía de la UNED, \\ Departamento de Geografía, Facultad de Geografía e Historia, \\ Universidad Nacional de Educación a Distancia, \\ C/ Senda del Rey, 7. 28040 Madrid \\ jfportela@geo.uned.es
}

\section{Eva María Martín Roda}

Grupo de Investigación Geografía, Paisaje y Tecnologías de la Información del Departamento de Geografía de la UNED, Departamento de Geografía, Facultad de Geografía e Historia,

Universidad Nacional de Educación a Distancia,

C/ Senda del Rey, 7. 28040 Madrid emartin@geo.uned.es

\section{María del Carmen Muguruza Cañas}

Grupo de Investigación Geografía, Paisaje y Tecnologías de la Información del Departamento de Geografía de la UNED, Departamento de Geografía, Facultad de Geografía e Historia,

Universidad Nacional de Educación a Distancia,

C/ Senda del Rey, 7. 28040 Madrid mmuguruza@geo.uned.es

\section{María Jesús Vidal Domínguez}

Grupo de Investigación GEOTAPLAN

del Departamento de Geografía de la UAM,

Departamento de Geografía, Facultad de Filosofía y Letras,

Universidad Autónoma de Madrid

C/ Francisco Tomás y Valiente, 1. 28049 Madrid

mariajesus.vidal@uam.es 
Resumen: La situación política actual se caracteriza por la fragmentación del voto que ha dado lugar al debilitamiento y, en algunos territorios, al fin del bipartidismo, como es el caso de la Comunidad de Madrid (CM). Las características mayorías absolutas del PP y del PSOE, han dejado paso a un abanico de gobierno más plural, donde la necesidad de los pactos es inevitable para lograr la gobernabilidad de un territorio.

Este trabajo analiza la reorganización y la situación actual del mapa político de la CM. Para ello se van a estudiar los resultados electorales de los comicios municipales de 2011, 2015 y 2019. Los datos utilizados para este trabajo son los que proporcionan el Ministerio del Interior y la Comunidad de Madrid, así como el Padrón Municipal de Habitantes del Instituto Nacional de Estadística. Tras una revisión bibliográfica de los estudios realizados sobre la Geografía Electoral, se han elaborado una serie de gráficos y mapas que sustentan las bases de esta investigación, y en los que el tamaño de los municipios es la variable más representativa.

El resultado es un escenario diverso en la CM entre 2011 y 2019. Se ha pasado de la hegemonía de los dos grandes partidos políticos, PP y PSOE, y en especial del PP, a un mapa con una variedad más amplia de opciones políticas, una mayor pluralidad política. Es decir, de un mapa monocolor a otro policromático en apenas ocho años y con notorias diferencias territoriales.

Palabras clave: Comunidad Autónoma de Madrid, Cambio en la tendencia del voto, Elecciones Municipales, Bipartidismo, Multipartidismo.

Abstract: The current political situation is characterized by a splitting of the vote that has led to the weakening and, in some territories, end of bipartisanship, as is the case of the Community of Madrid (CM). The characteristic absolute majorities of the PP and the PSOE have been replaced with a more colourful range of government, where the need for making deals across the aisle is a necessary step to govern.

This study analyses the reorganization and current situation of the CM's political map. To achieve this, the study will be based upon the electoral results of the municipal elections of 2011, 2015 and 2019. The data will be provided by the "Ministerio del interior" and the Community of Madrid, as well as the Register from the "Instituto Nacional de Estadística". After a bibliographic review of the studies carried out on Electoral Geography, a series of graphs and cartograms have been prepared that support the basis of this research, in which the municipalities' size is the most representative variable. The result is a diverse scenario in the CM between 2011 and 2019. The situation has switched between a bipartisan leadership shared between PP and PSOE (PP especially), to a figurative map with a wider variety of political options, a greater political plurality. In other words, from a single-color map to a multicolored one in just eight years and with notable territorial differences.

Keywords: Autonomous Community of Madrid, Change in voting trends, Municipal elections, Bipartisanship, Political diversity. 


\section{Introducción}

Este trabajo estudia el cambio en la tendencia en el voto en los municipios de la Comunidad de Madrid (CM), y la transformación de un sistema de representación política que ha estado tradicionalmente dominado por los dos partidos mayoritarios en España -Partido Popular (PP) y Partido Socialista Obrero Español (PSOE)-. Este bipartidismo deriva tanto de las peculiaridades históricas de España como de la transición política de la dictadura a la democracia, y del sistema de cálculo electoral de carácter proporcional utilizado y conocido como método D’Hondt, y que favorece la formación de mayorías.

A pesar del sistema de cálculo señalado, y de que está concebido para potenciar el bipartidismo, en las elecciones de 2015 el voto se fragmentó como consecuencia de la aparición en el espectro político de nuevos partidos y asociaciones políticas, lo que puede ser interpretado como un cambio sociológico de la población española. Esta transformación se produjo en el lapso transcurrido entre las elecciones municipales del año 2011 y las del 2015, y cuya tendencia se ha mantenido en las últimas elecciones municipales celebradas en mayo del año 2019 y que, aunque los dos grandes partidos siguen teniendo una representatividad importante sobre el resto de las formaciones políticas, se confirma la propensión a la composición de gobiernos municipales bicolor y multicolor, lo que es, sin duda, un cambio novedoso en el comportamiento electoral de la población española durante los últimos cuarenta años y que propiciaba la existencia de amplias mayorías y de una alternancia, casi exclusiva, entre los dos grandes partidos nacionales.

El objetivo principal de esta investigación es analizar la evolución del voto en las elecciones municipales de 2011, 2015 y 2019 en la CM para ver la evolución experimentada en el sistema dominante de alternancia bipartidista en estos años. Para ello se tienen que analizar una serie de hechos que resultan claves para comprender lo acontecido en la autonomía madrileña en este lapso temporal, focalizándonos principalmente en los siguientes puntos: la evolución de los dos grandes partidos políticos tradicionales que concentraban la mayor parte del voto y el reparto de los concejales en los gobiernos municipales, el paso del bipartidismo al pluripartidismo, y el análisis del comportamiento del voto en función del tamaño del municipio.

\subsection{La situación actual en la política española: el escaso papel de la geografía en los estudios electorales}

El estudio del espacio y de los fenómenos y acontecimientos que en él suceden, es una de las cuestiones prioritarias de las investigaciones en el campo de la Geografía. La Geografía Política, y en concreto la subdisciplina que se conoce como Geografía Electoral (Monzón, 2009), es una especialidad que se encarga de analizar los métodos, el comportamiento y los resultados de las elecciones desde la perspectiva espacio-temporal. 
El origen de la Geografía electoral se encuentra en Francia a finales del siglo XIX y comienzos del XX, durante el periodo de la Tercera República (Siegfried, 1913). Los geógrafos y politólogos de este periodo llamaron a la teoría de Siegfried "geometría de los círculos electorales". Este modelo se expandió a otros países de Europa y la producción científica en las décadas de los sesenta, setenta y ochenta del siglo XX fue muy fructífera. Algunos de los ejemplos más destacados se localizan en Gales (Cox, 1970), Alemania (Laux y Simms, 1973), Portugal (Cabral, 1977) o en España (Bosque, 1982).

La situación política de este periodo se encuentra muy distante de la actual, donde las transformaciones en los parlamentos europeos, y más recientemente en el caso de España, están cambiando de forma drástica y rápida a causa de la irrupción de nuevas fuerzas políticas. Esta situación ha provocado una grave crisis en el sistema bipartidista español que llevaba asentado desde el comienzo de la Democracia, y que algunos autores denominaban bipartidismo imperfecto (Llera, 1998; Blanco, 2016). En el caso de España, en la X Legislatura (2011-2015) el bipartidismo seguía arraigado con 185 diputados para el PP y 110 para el PSOE, entre ambos sumaban 295 diputados (84.28\% del total de la Cámara Alta). Sin embargo, en la corta XIII Legislatura (mayoseptiembre de 2019), el porcentaje de estos dos grupos políticos se redujo a 189 diputados, 54\% del total, mientras que en la Legislatura actual, la XIV, el bipartidismo se reforzó ligeramente respecto a la anterior hasta alcanzar, entre PSOE y PP, el 59,43\% de los Diputados. Aun así, un 25\% menos que en la X Legislatura.

La mayoría de los estudios sobre el fin del bipartidismo en España están de acuerdo en afirmar que es a partir de las elecciones de 2011, y en consonancia con la profunda crisis económica y social que se desencadenó en nuestro país, cuando el bipartidismo empezó a dar síntomas de agotamiento (Gil-Torres, 2018).

La situación política española que había venido marcada por una inercia bipartidista se ha visto interrumpida con la aparición de nuevas formaciones políticas tras las elecciones europeas de 2014, si bien Unión, Progreso y Democracia (UPyD) y los partidos nacionalistas habían tenido presencia en el parlamento español, no fue hasta 2015 cuando los partidos de Podemos y Ciudadanos (Cs) consiguen un número significativo de escaños, 69 y 40 respectivamente, y conjuntamente el 34,59\% de los votos, lo que supuso un importante cambio en la configuración del Congreso de los Diputados, así como a la hora de conformar gobierno.

El cambio se estaba produciendo, y el Movimiento del 15-M, también conocido como Movimiento de los Indignados, que tuvo su centro neurálgico en la Puerta del Sol de Madrid, fue el punto de partida de un malestar ciudadano ante la, según ellos, inadecuada respuesta dada por el bipartidismo a la fuerte crisis económica que asolaba el país y que tuvo importantes efectos en el imaginario popular y contribuyó a sentar las bases del cambio político que estaba produciéndose desde entonces (Sánchez, 2017). 
Se empezó a pensar que era posible lograr un gobierno más participativo, en el sentido de que comenzó a abrirse paso la idea de que los dos partidos mayoritarios ya no representaban al conjunto de la población del país, ni eran capaces de abordar las nuevas necesidades que habían surgido en un mundo globalizado, en el que los nuevos modelos económicos y productivos, las crisis cíclicas (comerciales, industriales y financieras como la de Lehman Brothers en EE.UU.), y afloradas en cualquier lugar del mundo podían afectar, y con toda virulencia, a cualquier nación.

A las elecciones municipales de 2015 siguieron, ese mismo año, las Elecciones Generales celebradas en el mes de diciembre, donde se pudo corroborar la nueva tendencia apuntada en los comicios anteriores con los dos nuevos partidos emergentes, configuradores de lo que ha dado en llamarse "nueva política" (Blanco, 2017), Podemos y Cs, sumaron en conjunto 109 diputados, una cifra algo inferior a la que consiguieron en las siguientes Elecciones Generales de junio de 2016 donde lograron 103 parlamentarios, aun así unos datos nada desdeñables. Esta cifra se ha incrementado tras los comicios de las generales de abril de 2019, al entrar en el escenario político un nuevo partido, VOX. Los tres conjuntamente lograron 123 representantes en el Congreso de los Diputados y el 40,5\% de los sufragios emitidos frente al 45,4\% que sumaron entre PSOE y PP. Esta situación ha permitido el cambio de un bipartidismo imperfecto a un multipartidismo fragmentado (Sánchez, 2017). Dentro de este contexto no podemos subestimar el papel que jugaron los partidos nacionalistas, tanto por su presencia en el Congreso de los Diputados, como en su papel decisivo en el apoyo a los dos grandes partidos nacionales a lo largo de los años y en diferentes gobiernos.

Esta posición no es nueva, sino que ya se ha manifestado en otros países, como, por ejemplo, Brasil (Scott, 1999), Bolivia (Archondo, 1999) o México (Sonnleitner, 2017). En este último caso se rastrean las dinámicas territoriales que han influido en la fragmentación partidista en este país entre 1991-2015. En los años noventa, la política mexicana se estructuraba en tres fuerzas mayoritarias y en la actualidad son casi seis, situación similar a lo que se está produciendo en España, así como en la Comunidad de Madrid.

Así pues, con matices y consideraciones que cabrían en un análisis de carácter más geopolítico que exclusivamente geográfico, podemos afirmar que en el año 2011 comienza la quiebra del sistema bipartidista que estaba instaurado en España desde las primeras Elecciones Generales democráticas de marzo de 1979 hasta las elecciones del año 2015, cuya secuencia había sido la alternancia entre el PSOE y el PP, que se repartían el control en municipios, autonomías y en la propia nación. Esta división del voto, además de enfrentar dos ideologías diferentes, socialdemócrata y liberalismo social, impedía llegar a acuerdos significativos en temas primordiales como educación, sanidad, leyes de dependencia, impuestos, ordenación del territorio, etc., pues en la UE, donde ha tomado carta de normalidad el denominado estado del bienestar, es difícil delimitar las diferencias entre ideologías opuestas, salvo en temas muy concretos, como los arriba citados, y que han sido utilizados profusamente de manera ideológica y partidista. 
Pero ¿qué ocurre en las elecciones municipales? El análisis de los resultados de las elecciones municipales ha sido poco investigado (Marschall et al., 2011). En el caso de la geografía electoral, pero también de la ciencia política, el interés por la política local es reducido frente a la atención otorgada a la política nacional (Kaufmann y Rodríguez, 2011).

La dimensión del ámbito local en todos los sentidos, tanto por número de municipios como por tamaño, no olvidemos que en el conjunto español hay 8116 municipios y en la Comunidad de Madrid 179, provoca un escenario territorial amplio, complejo y plural, que dificulta el desarrollo de este tipo de estudios, a pesar de ser significativos para el funcionamiento de los ayuntamientos y de las mancomunidades, así como para la ordenación del territorio. Como ejemplos se encuentran las investigaciones llevadas a cabo por Delgado (1999) donde analiza el comportamiento electoral de la población española, de forma agregada, desde las primeras elecciones democráticas de 1979 hasta las de 1995. El interés por este tipo de estudios se incrementa cuando se contempla la posibilidad de que los resultados obtenidos en ellos, puedan llegar a influir en el comportamiento de los electores a escala nacional de cara a la elección de un presidente de gobierno (Delgado, 2010), así como el impacto electoral que puede darse a escala local a la hora de votar a un partido u otro en función de la valoración que la población hace sobre un candidato a la alcaldía u otro, sin tener en cuenta la ideología política (Riera et al., 2017).

Los trabajos antes citados tienen un cariz eminentemente político, en el que la plasmación del voto en el espacio no es analizada de forma directa, cuando los resultados electorales tienen una clara influencia en los procesos espaciales tales como la creación de suelo, urbano y dotacional, los servicios, los planes de ordenación territorial y un largo etcétera, es por lo que consideramos necesario abordar el estudio de los resultados electorales desde la geografía, con la finalidad de precisar, espacialmente, las tendencias políticas de la población y, de esta manera, abrir nuevas líneas de estudio que permitan relacionar el territorio con otros indicadores sociales como renta, paro, servicios, conectividad, etc.

\section{Metodología}

En primer lugar realizaremos el estudio estadístico de los resultados electorales en cada municipio y en función de los datos oficiales existentes, determinaremos la composición política de los gobiernos municipales, para analizar cuestiones tales como los cambios acaecidos en los municipios, determinar si existe fidelidad, o no, en función del tamaño del municipio, con los partidos tradicionales, identificar si la fragmentación del voto es semejante en las pequeñas localidades y en las grandes; y en qué medida. 
También, para tener una rápida visualización de los datos, se realizará una cartografía que refleje los resultados del estudio estadístico.

Como fase previa de este trabajo, se realizó un exhaustivo análisis de la bibliografía existente, observando que, si bien, la geografía electoral había tenido una cierta importancia en los años 80 y 90 del siglo XX en los trabajos geográficos, este interés se había ido diluyendo paulatinamente. Por este motivo es por lo que decidimos abordar de nuevo el análisis de geografía electoral.

Desde el punto de vista de las fuentes estadísticas se consideraron varias, sopesando los pros y los contras de estas. En primer lugar, se consultó el Anuario estadístico del País, que recoge una importante información electoral. El problema que encontramos es que los datos ofrecidos están bastante elaborados, por lo que no era la fuente idónea al considerar que éramos nosotros los que deberíamos determinar los clústeres de agregación. La siguiente fuente analizada fueron los datos brindados por el Ministerio del Interior, donde hay series de los resultados electorales a todas las escalas espaciales. Nos enfrentamos con el problema de que no todos los datos podían ser descargados en una hoja de cálculo. La composición partidista municipal debía ser copiada manualmente, lo que podía generar algún tipo de error de transcripción.

Finalmente, se encuentra la información proporcionada por el Instituto de Estadística de la Comunidad de Madrid con datos accesibles mediante la descarga de una hoja de cálculo, para los comicios de 2011 y 2015, lo que eliminaba el error de transcripción. Por este motivo nos decantamos por esta última fuente para la realización del estudio estadístico, y emplear las otras dos como fuentes de verificación y apoyo.

Para el año 2019, y en el momento de redactar este trabajo, no había datos oficiales en ninguna de las fuentes antes citadas, pero hemos pensado que era importante contemplar la evolución del voto incluyendo el mayor número de información posible, se decidió acudir a fuentes provisionales. En ninguno de los organismos se encontraban los datos descargables en formato Excel, no obstante, en el Ministerio del Interior encontramos datos provisionales, por ello, y porque pensamos que el sesgo entre los datos provisionales y los definitivos no serán significativos para analizar la tendencia en el voto, decidimos utilizarlos. A este tenor, y para realizar el estudio teniendo en cuenta el número de habitantes de los municipios, se han utilizado los datos del Padrón Municipal de Habitantes del Instituto Nacional de Estadística (INE) a 1 de enero de 2018, y se han clasificado los municipios en intervalos según el tamaño de su población.

En la Comunidad de Madrid hay 179 municipios, incluida la ciudad de Madrid. Nuestro objeto de estudio es toda la CM, aunque hay que tener en cuenta que el Municipio de Madrid tiene unas peculiaridades especiales derivadas de su función de capital del estado y de su tamaño poblacional, y que, por lo tanto, el efecto capitalidad puede distorsionar los datos del conjunto. Aun así, es interesante ver lo acontecido en esta po- 
blación, compararlo con el resto de las entidades municipales y, con todo ello, ofrecer una visión global de lo ocurrido en la CM. La capital española representa el 48,81\% de la población de la región, y algo más de la mitad del electorado, en concreto, el 50,20\%.

A las elecciones municipales celebradas en el año 2011 concurrieron 194 partidos y en los comicios de 2015 y de 2019 el número de estos se incrementó en más de 40 nuevos partidos o agrupaciones políticas. Estas cifras hacen inviable cualquier estudio pormenorizado, pues la complejidad de la información que se puede extraer sería contraria a la claridad de la interpretación, a la comunicación y a su representación. Por ello se planteó la necesidad de agruparlos en clúster, para lo que procedimos a efectuar un estudio de los partidos que engrosaban esta cifra. Por un lado, entre cuatro y cinco partidos políticos eran fácilmente identificables, ya que eran los que se consideran como partidos tradicionales. Sin embargo, identificar el resto de los partidos era más complejo, pues en muchos casos era imposible, en función de las siglas de un grupo en concreto, identificar la tendencia ideológica, lo que nos generaban importantes dudas de agrupación. El ejemplo más claro es el caso de Unidas Podemos, ya que en algunos municipios aparecía con este nombre, y en otros casos concurría junto con otros partidos políticos o con agrupaciones de vecinos que presentaban nombres diversos.

Ante esta realidad se optó por indagar en la evolución de los partidos con mayor presencia y dispersión en el territorio, es decir, aquellos que han obtenido votos y representación en un mayor número de ayuntamientos. Así pues, para las elecciones de 2011 se han seleccionado el Partido Popular, el Partido Socialista Obrero Español, Izquierda Unida y Unión Progreso y Democracia, que conjuntamente concentraron el 92,60\% de los votos, el resto han sido incluidos en el clúster que hemos denominado Otros y que aglutinó el 7,40\% restante.

La situación ha variado en los comicios del año 2015 y 2019, con respecto a los celebrados en el año 2011. En el caso de las elecciones de 2015, además de los cuatro partidos mencionados anteriormente, se ha añadido Ciudadanos (Cs), y dentro del grupo Otros, que incrementa su porcentaje de votantes hasta el 30,46\% se ha incluido a Ahora Madrid, que solo se presentó en la capital madrileña, pero obtuvo un 16,56\% de los sufragios, más de la mitad de este grupo. En el año 2019 se ha añadido a VOX, que irrumpe con una importante representación municipal, y se ha eliminado a UPyD al desaparecer prácticamente del espectro político madrileño, así como a IU que se ha incluido en el apartado Otros, pues en algunas localidades se ha presentado en coalición con otros partidos políticos como con PODEMOS o EQUO. Dentro del grupo de Otros se incluye también Más Madrid, que viene a sustituir a Ahora Madrid y obtuvieron casi el 30\% de los votos. 


\section{Resultados. Un territorio en proceso de cambio: de la monotonía a la pluralidad política en la Comunidad de Madrid}

\subsection{Análisis en la evolución del voto municipal en los comicios de 2011, 2015 y 2019}

El panorama político de la CM ha experimentado importantes transformaciones en las tres últimas elecciones municipales (2011-2015-2019). La hegemonía de las dos grandes fuerzas políticas españolas, PP y PSOE, es cada vez más débil, pues el resultado conjunto de ambos en las Elecciones Municipales madrileñas de 2019 ha sido inferior al 50\%. No se puede decir que exista un partido que domine sobre el resto, pues se mueven en unas cifras porcentuales similares (ver Figura 1). En el caso del PP el 26,07\%, el PSOE con el 22,21\%, Cs el 17,80\% y el clúster denominado Otros el 25,78\%.

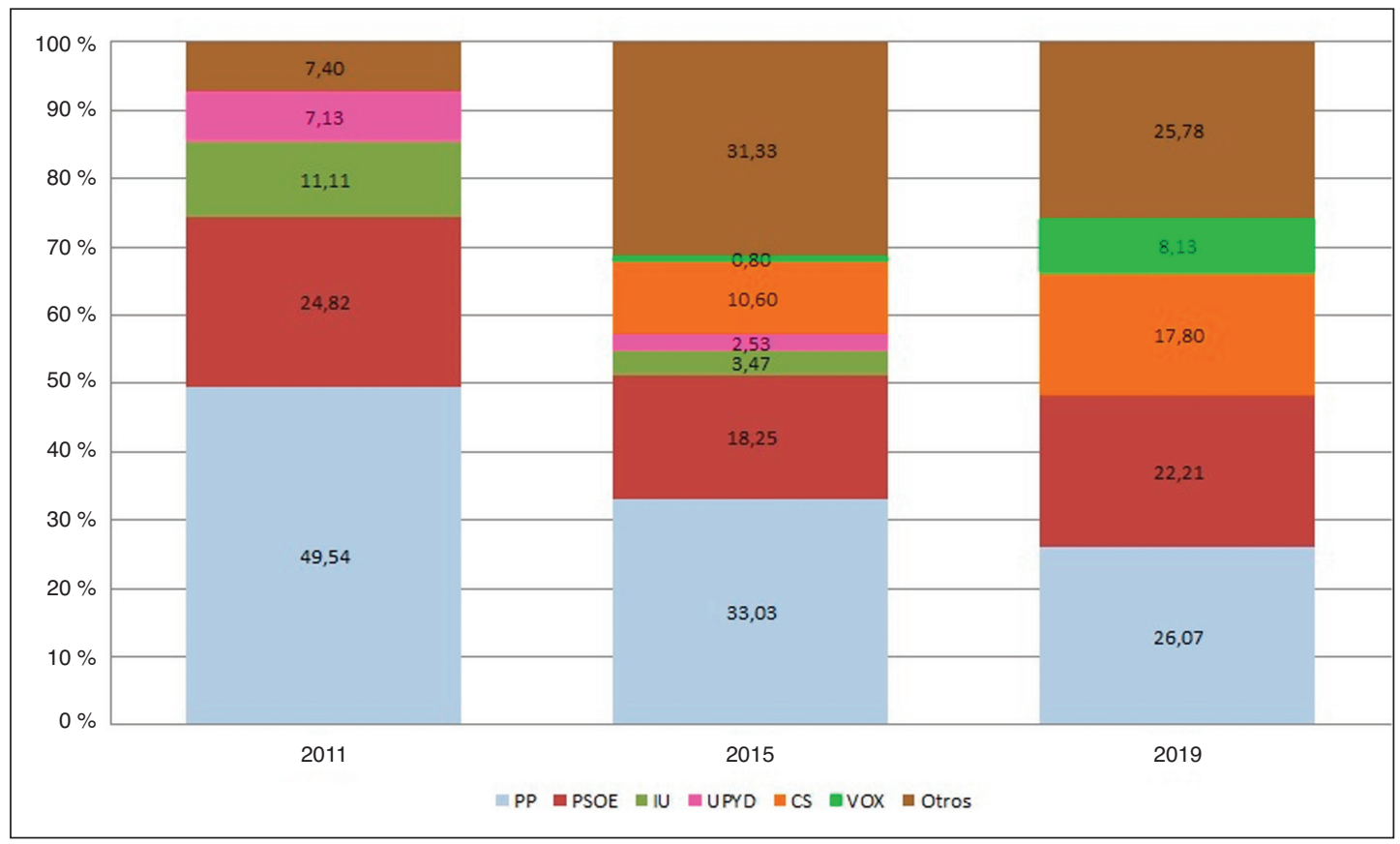

Figura 1. Evolución porcentual de votantes en las Elecciones Municipal en la CM en 2011, 2015 y 2019.

Fuente: Instituto de Estadística de la Comunidad de Madrid y Anuario El País. Elaboración propia.

Si bien, en lo concerniente al reparto de concejales, los dos grandes partidos se ven beneficiados y consiguen una mayor proporción de ediles $(61,04 \%)$ que de votos $(48,29 \%)$. La causa que lo explica es la fuerte fragmentación de los partidos políticos, muchos de 
ellos locales, y que en ocasiones no llegan al 5\% de sufragios necesarios para poder optar a tener un concejal (Método D'Hondt), por lo que, con esta situación, benefician a las listas más votadas.

En las elecciones municipales de 2011, el bipartidismo era evidente. En el caso de la CM la mayoría del Partido Popular fue aplastante, pues consiguió el mayor número de concejales, en total, entre todos los municipios de la CM y la propia capital, 1.207, lo que significó el 52,78\% del total (Ver Tabla 1). El PSOE quedó en segundo lugar con el $23,87 \%$. Entre ambos la cifra alcanzada fue de $76,65 \%$ de concejales, lo que se traduce en 1.753 representantes en los ayuntamientos de los 2.287 disponibles en la CM. Sin embargo, ya en el año 2011, se podía entrever un ligero descenso en cuanto a representantes municipales de estos dos grandes partidos políticos, con respecto a las elecciones municipales del año 2007, cuando consiguieron el 79,66\% de los concejales, es decir, un 3,01\% menos.

Tabla 1. Distribución de concejales por partidos políticos en las elecciones municipales de 2011, 2015 y 2019 en los municipios de la Comunidad de Madrid

\begin{tabular}{|c|c|c|c|c|c|c|}
\hline & \multicolumn{6}{|c|}{ CONCEJALES } \\
\hline & 2011 & Porcentaje (\%) & 2015 & Porcentaje (\%) & 2019 & Porcentaje (\%) \\
\hline Partido Popular (PP) & 1207 & 52,78 & 919 & 39,87 & 762 & 32,87 \\
\hline $\begin{array}{l}\text { Partido Socialista } \\
\text { Obrero Español (PSOE) }\end{array}$ & 546 & 23,87 & 521 & 22,60 & 653 & 28,17 \\
\hline Izquierda Unida (IU) & 178 & 7,78 & 151 & 6,55 & 64 & 2,76 \\
\hline $\begin{array}{l}\text { Unión Progreso y } \\
\text { Democracia (UPYD) }\end{array}$ & 64 & 2,80 & 17 & 0,74 & 1 & 0,04 \\
\hline Ciudadanos (Cs) & 0 & 0,00 & 137 & 5,94 & 257 & 11,09 \\
\hline Otros & 292 & 12,77 & 560 & 24,30 & 581 & 25,06 \\
\hline Total & 2287 & 100,00 & 2305 & 100,00 & 2318 & 100,00 \\
\hline Suma PP y PSOE & 1753 & 76,65 & 1440 & 62,47 & 1415 & 61,04 \\
\hline $\begin{array}{l}\text { Suma resto partidos } \\
\text { y OTROS }\end{array}$ & 534 & 23,35 & 865 & 37,53 & 903 & 38,96 \\
\hline
\end{tabular}

Fuente: Instituto de Estadística de la Comunidad de Madrid y Ministerio de Interior. Elaboración propia. 
Los resultados en los comicios locales auguraban el dominio del PP en las Elecciones Generales de 2011, y así fue. Prueba de ello es la mayoría absoluta que obtuvo, al conseguir 186 diputados y un 44,65\% del total del voto emitido. Estos resultados suelen ser considerados como unas primarias de cara a las elecciones generales al reflejar las preferencias políticas de los votantes (Riera et al., 2016).

Sin embargo, desde entonces, PP y PSOE han perdido representatividad en el panorama político, tanto nacional como local. Los datos de las elecciones de 2015 reflejan el cambio en el ciclo político, y en la sociología de la población, que se venía gestando y reforzaban la hipótesis, que todo el mundo consideraba plausible, del fin del sistema de partidos hegemónicos y del comienzo de la diversificación política. Esta situación implicaría un cambio importante en las actitudes partidistas e ideológicas, obligando a los líderes de los diferentes partidos políticos a llegar a acuerdos de gobierno en temas fundamentales, que les diese estabilidad y continuidad temporal, y ayudase a la gobernabilidad del territorio.

En las elecciones municipales de 2015 en la CM, tal y como preveían las encuestas, los dos partidos que conforman el bipartidismo obtuvieron el 62,47\% de los concejales, en torno a un 14\% menos que en los comicios de 2011, y un 17,19\% menos respecto a los comicios de 2007. Una tendencia de decrecimiento que se lleva manteniendo durante los últimos cuatro procesos electorales, aunque es entre 2011 y 2015 cuando se intensifica, dando lugar a una clara fragmentación del bipartidismo y la irrupción de los nuevos actores políticos que ya hemos citado.

En las elecciones del año 2019 se produce un ligero descenso del 1,4\% respecto a las elecciones de 2015, representando las dos fuerzas mayoritarias el 61,07\%, unas cifras que, en relación con lo acontecido en el año 2015, parecen indicar cierta estabilización en el cómputo global de la representación de ambos partidos. En estos últimos comicios, año 2019, el PP experimenta un nuevo retroceso, con la pérdida de un 7\% de los concejales, mientras que en el PSOE se ha producido un repunte del 5,57\% más con respecto a las elecciones municipales del año 2015, cifras semejantes a las logradas en las Elecciones Generales de abril de 2019 donde el PSOE ganó los comicios y el PP se hundió.

La tabla 1 muestra información relevante sobre los otros actores presentes en el escenario político, configurando un elenco más amplio y plural. Izquierda Unida pierde el $1,23 \%$ de sus concejales en las elecciones municipales de 2015, pasando de 178 a 151, y en las de 2019 disminuye hasta los 64. Si bien, es cierto que, en el caso de IU, los datos de la representación conseguida por este partido son difíciles de comparar al haberse presentado, en función del municipio de que se trate, o bien sólo o bien en confluencia con Unidas Podemos, con otros partidos políticos e incluso con asociaciones vecinales.

Realmente llamativo es el caso de UPYD, que prácticamente desaparece pasando de los 64 concejales que consiguió en las elecciones del año 2011, a 17 en el sufragio 
del año 2015, y a uno en los comicios de 2019. Frente a la debacle del partido que fundó Rosa Díez hallamos el florecimiento del partido de Cs, que logró en las primeras elecciones municipales a las que se presentó en la CM, las de 2015, un total de 137 ediles, y 257 concejales en las celebradas en 2019, aglutinando una parte importante del voto del partido magenta y una parte del PP.

En el clúster que hemos designado como Otros, aparecen partidos locales, asociaciones y agrupaciones municipales, y creemos que también están incluidas algunas de las confluencias que presuponemos afines a Unidas Podemos, pero cuya filiación política no hemos podido comprobar, ya que en su propaganda electoral no quedaba explícita su ideología. Este conjunto, prácticamente ha duplicado sus resultados, pues ha pasado del 12,77\% de los concejales en el año 2011 al 24,30\% en las elecciones de 2015. En el año 2019, las cifras han sido muy similares respecto a los comicios de 2015. La importante representatividad de este grupo de Otros, ha sido posible gracias a la obtención de los buenos resultados en 2015 en localidades de cierta entidad poblacional como Parla ( $45,17 \%$ de los votos), Leganés (42,72\%) o Aranjuez (31,32\%), o la victoria que tuvo el partido político Más Madrid (30,94\% del voto) en la ciudad de Madrid en las elecciones de 2019.

Los resultados que venimos analizando confirman, para la CM, un cambio en la tendencia del voto, transitando los votantes desde el bipartidismo pretérito a un abanico de posibles opciones para conformar gobiernos, que establecen una realidad socio territorial diferente y, como consecuencia, un mapa electoral cambiante que pasa del casi azul monocolor de 2011 a uno multicolor en 2019.

\subsection{La configuración del territorio en la $\mathrm{CM}$ : el nuevo mapa político (2011-2019)}

Para analizar lo sucedido en los municipios de la CM, se van a tener en cuenta los resultados obtenidos por tamaño de población, teniendo en cuenta dos variables. Por un lado, el porcentaje de votos a los partidos políticos, y por otro el número de concejales conseguidos en cada uno de ellos.

En los comicios del 2011 el PP fue el partido más votado en todos los intervalos poblaciones, consiguiendo más del 50\% de los concejales y alrededor del 50\% del voto total en todos ellos. Ganó en 148 municipios de los 179 que tienen la CM, de los cuales, en 89, obtuvo más del 50\% de los votos (ver Figura 2). El PSOE se alzó con 15 mayorías absolutas, IU fue la fuerza más votada en tres localidades, y el grupo denominado Otros se alzó con la victoria en 13 municipios. El resultado global fue un mapa prácticamente monocolor, con un claro dominio del PP en la capital madrileña, en su corona metropolitana, la sierra y el sur de la comunidad. 


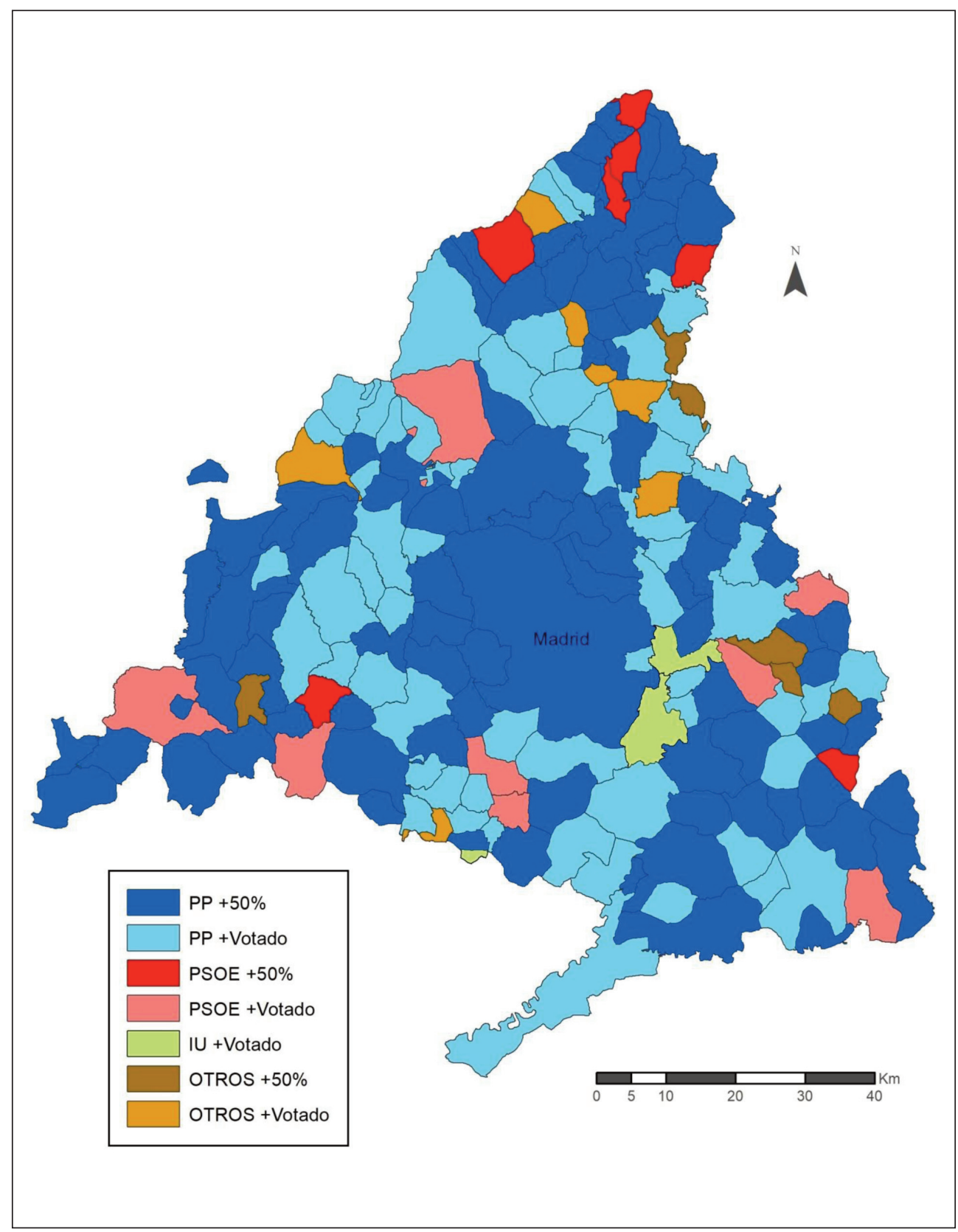

Figura 2. Partidos con más del 50\% de los votos y más votados en 2011.

Fuente: Instituto de Estadística de la Comunidad de Madrid 2011. Elaboración propia. 
Es en los municipios de menos de 500 habitantes donde el PP obtuvo los mejores resultados con el 51,09\% de los votos y el 57,75\% de los concejales (Ver Tabla 2). Este grupo de municipios, un total de 28 , que representan tan solo el 0,12\% del total de votantes, pero donde se reparten el 6,21\% de los concejales, cifras que indican el peso significativo de estas pequeñas poblaciones, y que se ha dejado patente en la campaña electoral de las Elecciones Generales de abril de 2019, donde los partidos políticos han salido "a la caza" del voto rural que ha resultado decisivo en la obtención del último diputado en algunas circunscripciones. Hay que añadir que, en estos espacios, la fidelidad del voto a determinados candidatos de partidos tradicionales es muy significativa, y es más complicada la entrada de nuevos partidos políticos ante la ausencia de candidatos que quieran concurrir a las elecciones y con siglas nuevas.

Tabla 2. Resultados elecciones municipales 2011 por tamaño del municipio (votos y concejales)

\begin{tabular}{|l|l|r|r|r|r|r|r|}
\hline \multicolumn{2}{|c|}{} & $\begin{array}{c}\text { Total } \\
\text { municipios }\end{array}$ & $\begin{array}{c}\text { Menos } \\
\text { de } 500\end{array}$ & $\begin{array}{c}\text { De } 500 \\
\text { a } 10 \text { 000 }\end{array}$ & $\begin{array}{c}\text { De 10 000 } \\
\text { a 50 000 }\end{array}$ & $\begin{array}{c}\text { De 50 000 } \\
\text { a } 100 \text { 000 }\end{array}$ & $\begin{array}{c}\text { Más de } \\
100 \text { 000 }\end{array}$ \\
\hline \multirow{2}{*}{ Total municipios } & $\mathbf{1 7 9}$ & $\mathbf{2 8}$ & $\mathbf{1 0 3}$ & $\mathbf{2 8}$ & $\mathbf{1 0}$ & $\mathbf{1 0}$ \\
\hline \multirow{2}{*}{ PP } & Votos & 1447701 & 1798 & 82293 & 134272 & 157508 & 1071830 \\
\cline { 2 - 9 } & Concejales & 1207 & 82 & 568 & 266 & 134 & 157 \\
\hline \multirow{2}{*}{ PSOE } & Votos & 725205 & 740 & 43587 & 51187 & 63.624 & 566067 \\
\cline { 2 - 9 } & Concejales & 546 & 30 & 275 & 98 & 54 & 89 \\
\hline \multirow{3}{*}{ IU } & Votos & 324776 & 182 & 15110 & 31702 & 42.258 & 235524 \\
\cline { 2 - 9 } & Concejales & 178 & 3 & 61 & 49 & 33 & 32 \\
\hline \multirow{2}{*}{ UPYD } & Votos & 208213 & 0 & 1545 & 17150 & 25.838 & 163680 \\
\cline { 2 - 9 } & Concejales & 64 & 0 & 6 & 25 & 16 & 17 \\
\hline \multirow{2}{*}{ Otros } & Votos & 216093 & 799 & 31689 & 45641 & 28.350 & 109614 \\
\cline { 2 - 9 } & Concejales & 292 & 27 & 165 & 82 & 13 & 5 \\
\hline
\end{tabular}

Fuente: Instituto de Estadística de la Comunidad de Madrid 2011. Elaboración propia.

En el resto de los tramos de municipios, el PP se acercó al 50\% de los votos. El resultado del voto no varió mucho en función de su tamaño. En las localidades de entre 500 y 10000 habitantes obtuvo el 47,23\% del voto y el 52,88\% de los concejales. Debemos destacar que, en este intervalo de población, en su mayoría, municipalidades de carácter rural, se concentraba tan solo el 5,96\% de los sufragios para el año 2011, pero que, sin embargo, este porcentaje de votantes representaba el $47 \%$ de los conce- 
jales totales. Estos municipios van a ser vitales para los partidos políticos, pues concentran una cuota de poder muy relevante de cara a la configuración de los ayuntamientos.

Respecto a los municipios de más de 100000 habitantes, en total diez, aglutinan el $73,47 \%$ de los votos de la CM y el 12,12\% de los concejales (300 en total). El número electo de concejales es inferior a los que suman el total de municipios de entre $500 \mathrm{y}$ 10000 habitantes (1075 concejales y 5,96\% de los votos), pero en esta ocasión gana relevancia el peso de los votantes, pues concentran casi a las tres cuartas partes del electorado, además de ser las unidades de población más grandes, incluida la ciudad de Madrid. Aunque el número de concejales sea menor, el poder político que acaparan a la hora de tomar decisiones y marcar tendencias políticas es decisivo, y van a ser claves para la conformación del gobierno regional.

Cuatro años después, en las Elecciones Municipales de 2015, el panorama cambió, aunque PP y PSOE fueron, de nuevo, los más votados, perdieron representatividad en los gobiernos municipales. A pesar de estos cambios, el PP fue la fuerza política más votada en un total de 118 municipios (ver Figura 3), pero con menos apoyos que en los comicios de 2011 al perder un 20\% de las localidades. El PSOE logra 29 victorias, casi el doble que, en los comicios de 2011, IU gana las elecciones en cuatro y Cs en tres municipios, y el clúster denominado Otros se alza como la fuerza más votada en un total de 25 localidades. Estos resultados, dan lugar a un mapa diverso al de 2011, con un territorio con mayor pluralidad política y más contrastes espaciales. Sin embargo, es en las entidades de población de mayor tamaño donde se vislumbra el cambio de forma más evidente, como también lo es la pérdida de poder de los grandes partidos políticos, que copaban, hasta estas últimas elecciones, la mayor parte de los ayuntamientos.

Las heridas al bipartidismo se observan de forma más clara en los municipios que tienen más de 10000 habitantes (ver Tabla 3). Es en estos donde los nuevos partidos políticos han entrado con más fuerza, ganando y gobernando en ayuntamientos que, hasta la fecha, la mayoría de ellos estaban bajo mandato del PP o del PSOE. En los últimos tres tramos de municipios (10 000-50 000, 50 000-100 000 y más de 100000 habitantes), el PP pierde, aproximadamente, el 30\% de concejales y el $28 \%$ de votantes, mientras que el PSOE pierde el 13\% de los concejales y el 22\% de los votos. Los máximos beneficiados son el grupo Otros que triplica su representación con casi 200 nuevos concejales y más de 700000 votos, y Cs que entra en la política madrileña con 137 concejales y 321000 votantes. Estos valores demuestran la disminución de poder del gran bloque que conforman PP y PSOE. Esta fragmentación ha obligado a la aparición de una política de pactos y alianzas que hasta la fecha había tenido un escaso papel, pues las mayorías absolutas eran más comunes, y en caso de necesitarlo, se solía pactar con otra formación, pero en raras ocasiones era necesario el apoyo de dos o más grupos políticos para conformar el gobierno de un ayuntamiento. 


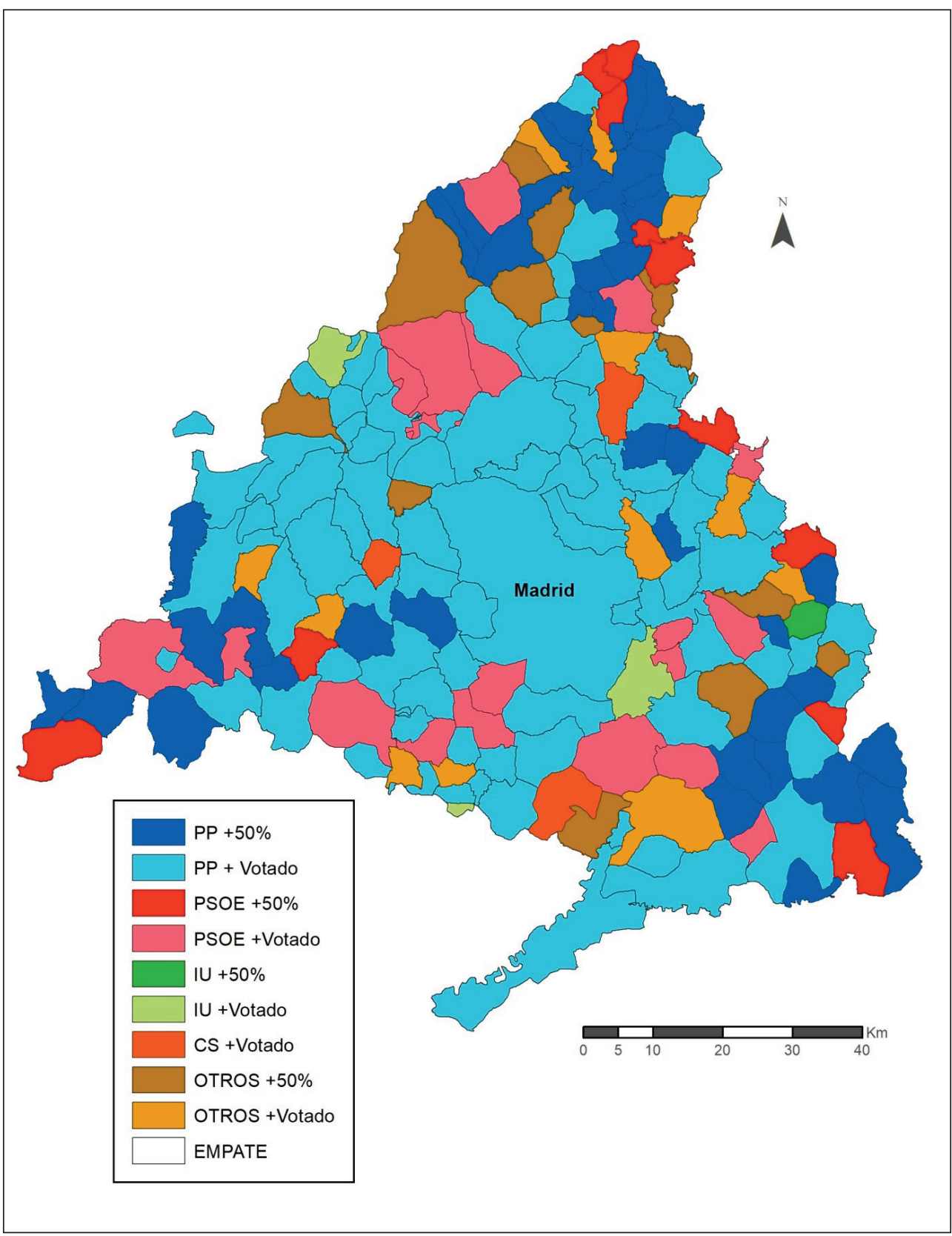

Figura 3. Partidos con más del $50 \%$ de los votos y más votados en 2015.

Fuente: Instituto de Estadística de la Comunidad de Madrid 2015. Elaboración propia. 
Tabla 3. Resultados elecciones municipales 2015 por tamaño del municipio (votos y concejales)

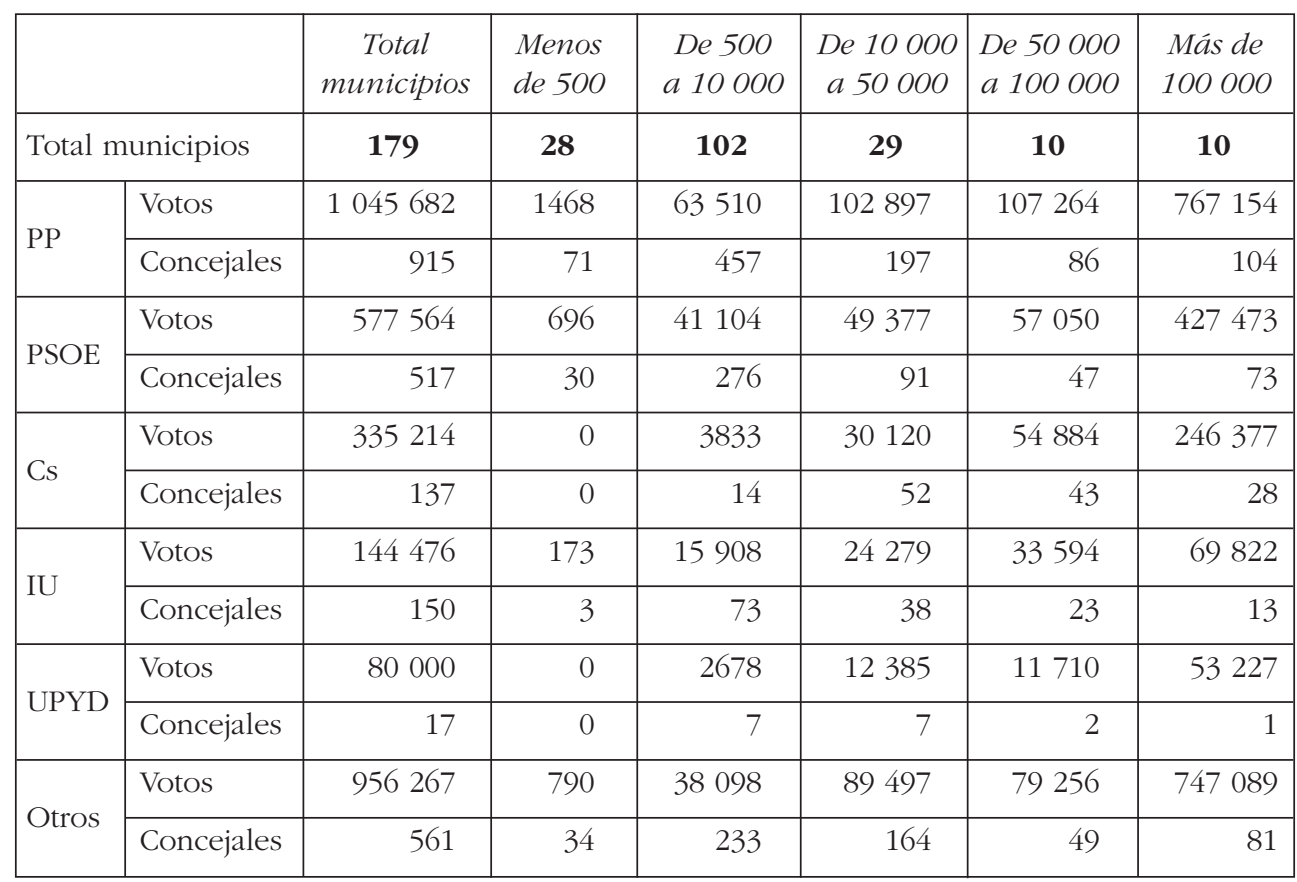

Fuente: Instituto de Estadística de la Comunidad de Madrid 2015. Elaboración propia.

Ejemplo significativo de esta ruptura del bipartidismo es la ciudad de Madrid, donde el PP, fue la fuerza más votada en 2015 con el 34,31\% de votos y 21 concejales, pero perdió el gobierno a favor de Ahora Madrid. Este partido consiguió el 31,61\% de los sufragios y 20 concejales, y gracias al pacto de investidura que realiza con el PSOE, que consiguió el 15,16\% de los votos y 9 concejales, logró el poder del ayuntamiento. Si se comparan estas cifras con los resultados de la capital madrileña en los comicios de 2011, se ve el notable desgaste del PP y del PSOE, con un 14,47\% menos de votantes y la pérdida de 10 concejales en el caso del primero, y del 8,34\% de los votos y 6 concejales en el segundo. La suma de ambos partidos se reduce de un $72,28 \%$ del voto y el $80,70 \%$ de los concejales, a un porcentaje de votantes del $49,48 \%$ pero que supone el 52,63\% de los representantes en el consistorio. Junto a Ahora Madrid se produce la irrupción, en la corporación municipal, de Ciudadanos con siete concejales, en parte a costa de UPYD que pierde los cinco ediles que consiguió en 2011, y la salida también de IU que se deja los seis concejales a favor de Ahora Madrid. Este panorama refleja un cambio de tendencia, no solo en la capital, sino en numerosos municipios, en especial de la primera y segunda corona metropolitana frente a las localidades del norte de Madrid que se mantienen más fieles a los partidos tradicionales. 
Esta realidad multipartidista se ha consolidado y reforzado en las elecciones municipales de abril de 2019 con una mayor representatividad de formaciones políticas muy diversas. Espacialmente, el mapa electoral de 2019 (ver Figura 4) nada tiene que ver con el de 2011, pues ha sufrido significativas trasformaciones, del casi monocolor al multicolor. El PP fue la fuerza más votada en 90 municipios, en 34 de ellos con más del 50\% de los votos, unos resultados muy alejados de los que tuvo en 2011. El PSOE experimenta un importante crecimiento convirtiéndose en el partido más votado en 53 ayuntamientos, beneficiándose del desgaste del PP a escala nacional, así como por las diversas tramas de corrupción que se están investigando y que afectan al PP madrileño. Finalmente, el resto de las fuerzas políticas (Cs, IU y Otros) fueron las más votadas en 36 de los 179 municipios, entre ellos, la ciudad de Madrid. Una realidad que, a pesar de la pluralidad, permite observar como el PP tiene su núcleo de apoyos en los municipios del norte, donde se ubican, principalmente, las empresas relacionadas con el terciario avanzado, tecnológicas y financieras, y la corona metropolitana oeste de elevado poder adquisitivo, frente al PSOE que tiene mayor representación en los municipios del cinturón industrial madrileño tanto en el sur de la comunidad como en torno a la carretera Nacional II.

A pesar de la caída del PP, en las Elecciones Municipales de 2019, sigue siendo la fuerza más votada con un 24,69\% de los votos frente al 49,54\% que obtuvo en los comienzos de 2011, es decir, ha perdido la mitad de su electorado en apenas 8 años y 446 concejales hasta lograr los 761 en los últimos comicios (ver Tabla 4). Probablemente, estos votos perdidos por el PP han ido a parar a dos formaciones políticas que han pasado de no tener representación, a aglutinar de forma conjunta el 24,71\% de los votos (Cs el 16,96\% y VOX el 7,75\%), es decir, prácticamente el porcentaje de votos que ha perdido el PP (24,85\%).

El PSOE también pierde electores entre el 2011 y el 2015, pero consigue remontar en las elecciones de 2019 con 100000 nuevos votantes acercándose al PP, por lo que la pérdida es menos severa, un 6,5\% en estos ocho años. Una parte importante de estos votantes procede del grupo Otros (PODEMOS, IU y otros), que en 2019 retroceden respecto a los resultados de 2015. Hay que tener en cuenta que, en el año 2015, IU y Otros se han analizado por separado, por lo que, en ese año su suma conjunta era de 1,1 millones de votantes, mientras que en 2019 apenas alcanzó el millón de votantes (ver Tabla 4), unos 150000 votos menos entre los dos últimos comicios.

Al igual que en los años anteriores, en los municipios de menos de 500 habitantes, el PP es la fuerza más votada y consigue más de la mitad de los concejales disponibles. La fragmentación del voto en los pequeños municipios es menor que en los grandes, y la irrupción de los nuevos partidos en estas entidades de población ha sido menor. Las listas tradicionales, PP y PSOE, son las más habituales, a lo que hay que sumar la mayor fidelidad del electorado, más envejecido en estos municipios más pequeños que en los del periurbano de Madrid. 


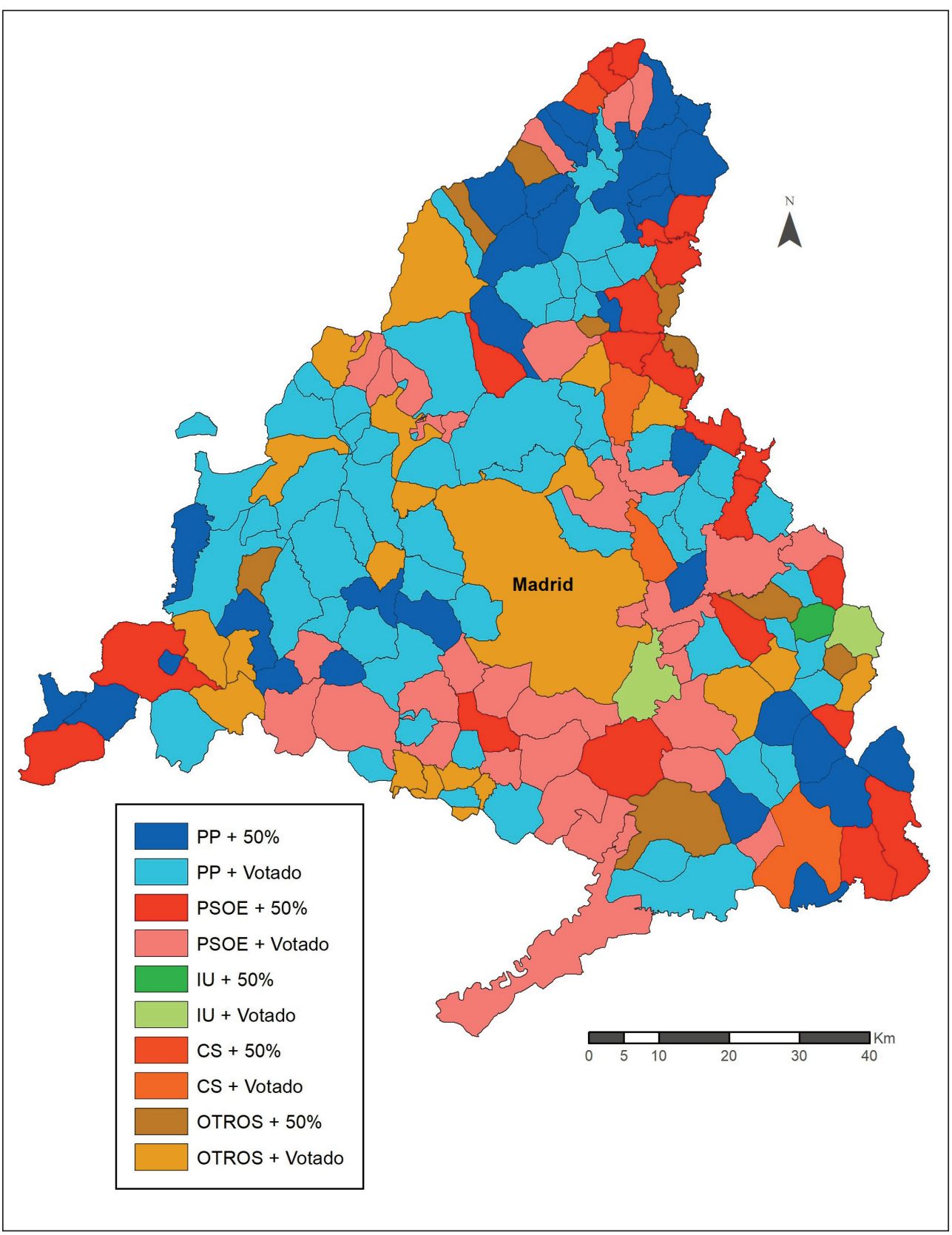

Figura 4. Partidos con más del 50\% de los votos y más votados en 2019. Fuente: Instituto de Estadística de la Comunidad de Madrid. Elaboración propia. 
Tabla 4. Resultados elecciones municipales 2019 por tamaño del municipio (votos y concejales)

\begin{tabular}{|l|l|r|r|r|r|r|r|}
\hline \multicolumn{2}{|c|}{} & $\begin{array}{c}\text { Total } \\
\text { municipios }\end{array}$ & $\begin{array}{c}\text { Menos } \\
\text { de } 500\end{array}$ & $\begin{array}{c}\text { De } 500 \\
\text { a } 10 \text { 000 }\end{array}$ & $\begin{array}{r}\text { De 10 000 } \\
\text { a 50 000 }\end{array}$ & $\begin{array}{c}\text { De 50 000 } \\
\text { a 100 000 }\end{array}$ & $\begin{array}{c}\text { Más de } \\
100 \text { 000 }\end{array}$ \\
\hline \multirow{2}{*}{ Total municipios } & $\mathbf{1 7 9}$ & $\mathbf{2 8}$ & $\mathbf{1 0 0}$ & $\mathbf{2 9}$ & $\mathbf{1 2}$ & $\mathbf{1 0}$ \\
\hline \multirow{2}{*}{ PP } & Votos & 795816 & 1646 & 56966 & 76241 & 113332 & 547631 \\
\cline { 2 - 9 } & Concejales & 761 & 71 & 375 & 147 & 90 & 78 \\
\hline \multirow{3}{*}{ PSOE } & Votos & 678231 & 832 & 49949 & 64273 & 95967 & 467210 \\
\cline { 2 - 9 } & Concejales & 652 & 33 & 308 & 128 & 83 & 100 \\
\hline \multirow{3}{*}{ Cs } & Votos & 546719 & 220 & 15779 & 39999 & 76053 & 414668 \\
\cline { 2 - 9 } & Concejales & 254 & 7 & 66 & 75 & 58 & 48 \\
\hline \multirow{2}{*}{ VOX } & Votos & 249824 & 0 & 10622 & 26498 & 40226 & 172478 \\
\cline { 2 - 9 } & Concejales & 140 & 0 & 40 & 51 & 29 & 20 \\
\hline \multirow{2}{*}{ Otros } & Votos & 951929 & 551 & 44401 & 82762 & 91375 & 732840 \\
\cline { 2 - 8 } & Concejales & 507 & 22 & 248 & 143 & 40 & 54 \\
\hline
\end{tabular}

Fuente: Instituto de Estadística de la Comunidad de Madrid. Elaboración propia.

Respecto a los municipios de más de 100000 habitantes, el PSOE adquiere un mayor número de concejales que el PP, a pesar de obtener más votos este último. Esto se debe a que, en este tipo de municipios, si no tenemos en cuenta a la ciudad de Madrid, donde se reparten un total de 57 concejales, el PSOE logra más votantes que el PP, 243628 del primero frente a los 152923 del segundo. Los socialistas consiguen mayorías en siete de las nueve localidades en este intervalo de población como por ejemplo Alcalá de Henares, Alcorcón o Fuenlabrada, frente a dos de los populares, en Alcobendas y Torrejón de Ardoz, por este motivo, el número de concejales obtenidos es mayor consiguiendo 92 actas el PSOE y 63 actas el PP.

En el resto de los intervalos, el PP consigue el mayor número de concejales, con diferencias entre el resto de los partidos cada vez más estrechas respecto a años anteriores. Sin embargo, el grupo Otros le supera en votantes en el tramo de 10000 a 50000 habitantes en más de 6000 sufragios, aunque obtiene 4 concejales menos, 143 frente a 147 del PP.

Significativa es la victoria del grupo de Otros en algunas localidades, es especial en la propia ciudad de Madrid, que pasa a ser una isla que ejerce de elemento separador entre el norte de la Comunidad Autónoma, con mayor peso del PP (rentas per cápita más altas), frente al sur con una mayor representatividad del PSOE (rentas per cápita más bajas). 


\section{Discusión}

En España y en concreto en la CM, en este momento de cambios políticos tan importantes, es cada vez más necesario el papel de la Geografía como una disciplina que ayude a ver, interpretar y entender lo que está pasando en el territorio. La política influye directamente en lo que sucede en la sociedad, tanto en las personas como en su lugar de residencia. Sin embargo, los estudios en Geografía Electoral no son frecuentes, y ha sido la Sociología, el Derecho y las Ciencias Políticas quienes se han ocupado en las últimas décadas de explicar el comportamiento de la sociedad, relegándose el componente espacial. Por ello, es necesario que la Geografía recupere el papel que tuvo en este tipo de estudios, y muestre y explique cómo el cambio actual de la situación que se está viviendo está influyendo en la organización del territorio.

Que el sistema bipartidista se encuentra en crisis es evidente, y varios investigadores lo han reflejado en sus trabajos (Blanco, 2016; Sánchez, 2017; Gil, 2018). El análisis que se ha realizado sobre las tres últimas elecciones municipales nos permite apoyar esta idea con datos concretos y recientes en el caso de la CM. Como han comentado estos autores, es en las Elecciones Municipales de 2011 cuando comienza a forjarse todo este movimiento de cambio y descontento por parte de la población, y entra en escena el multipartidismo y la fragmentación política del territorio.

El multipartidismo ofrece al elector una mayor oferta donde elegir el partido político que mejor se identifique con sus ideas e intereses. Prueba de ello es que los nuevos partidos políticos, en unos casos se han convertido en la llave de gobierno de numerosos ayuntamientos, y en otros casos han conseguido gobernar los ayuntamientos gracias a una serie de pactos y coaliciones. Según De Montalvo (2015) es un elemento positivo, pues permiten integrar personas e ideas diversas en un gobierno, y el diálogo, la negociación, la flexibilidad y la adaptabilidad se convierte en una herramienta esencial para conseguir la gobernabilidad (Sodaro, 2006). De Montalvo también añade que esto supone un esfuerzo y tiempo en la toma de decisiones y, que en ocasiones da lugar a gobiernos débiles. Efectivamente, estamos de acuerdo en que la política de pactos requiere de diálogo y de tiempo, y que otorga una mayor pluralidad en los consistorios o en los gobiernos regionales. Esto es positivo, pues en ocasiones evita malas decisiones llevadas a cabo por gobiernos con mayorías absolutas que no tienen en cuenta al resto de grupos políticos que forman las corporaciones municipales, pero también hay situaciones en las que la gobernabilidad es insostenible y las instituciones públicas quedan casi paralizadas. Respecto a la formación de gobiernos débiles es cuestionable, pues tenemos ejemplos de gobiernos regionales y municipales donde han tenido que pactar dos, tres y hasta cuatro fuerzas políticas y han conseguido, no solo acabar la legislatura, sino que, en algunos casos, han repetido la coalición. También hay que decir que ha habido malas experiencias y se han producido rupturas en algunas coaliciones originando parálisis en diferentes proyectos territoriales urbanos y sociales. 
Desde el punto de vista espacial, la plasmación en el mapa de los resultados electorales evidencia la fragmentación ideológica de la población, que está relacionada con factores socioeconómicos, como la edad de la población, formación, niveles salariales lo que es clara muestra de la dicotomía en la funcionalidad del territorio madrileño.

\section{Conclusiones}

Las Elecciones Municipales celebradas en 2011, 2015 y 2019, han dado lugar a un panorama político en la CM muy contrastado y diverso. Los cambios espaciales han configurado un nuevo mapa político en esta región, donde el PP ha sido el mayor perjudicado con la pérdida de votantes y de representantes municipales (en torno a la mitad de su electorado), así como del PSOE, que, a pesar de perder votantes y concejales, ha conseguido un mayor número de victorias en los ayuntamientos. El debilitamiento del bipartidismo es un hecho claro, y ha tenido lugar a favor de otras fuerzas políticas, como por ejemplo Cs, VOX y el clúster Otros (Más Madrid, Unidas Podemos y numerosos partidos locales y vecinales), que consiguen aumentar su peso político en la escena municipal, configurando un territorio diverso, plural y, en ocasiones, complejo de gobernar.

Los partidos políticos que han conseguido más del 50\% de los votos han disminuido considerablemente, este porcentaje se alcanzó en 102 municipios en las elecciones de 2011 frente a los 66 en 2019. Dentro de este grupo de entidades de población que sobrepasan el 50\% de los votantes, se encuentran, principalmente, los municipios con menor población donde se reparten menos concejales, entre 3 y 9, como por ejemplo Robledillo de la Jara (3), Berzosa del Lozoya (5), Brea de Tajo (7) o Valdeavero (9). Espacios donde se han presentado menos candidaturas, en la mayor parte de las ocasiones tan solo dos, por lo que es más fácil lograr estos porcentajes de votos. Sin embargo, hay que añadir algunos ejemplos de entidades de mayor población, que también han conseguido el 50\% de los votos como por ejemplo el PP en Torrejón de Ardoz (57,54\%), excepción entre los municipios situados a lo largo de la carretera Nacional II.

Si bien hay que decir, que, para obtener mayoría absoluta de concejales, no es necesario llegar al 50\% de los sufragios, pues debido al método D'Hondt, y a la fragmentación del voto, hay partidos políticos que no han alcanzado el 50\% de los votos, pero han conseguido mayoría absoluta de concejales. Como ejemplo se encuentran Arganda del Rey donde el PSOE fue la fuerza más votada con el 45,51\% de los votos y consiguió 13 de los 25 concejales, o Torrelaguna donde este mismo partido obtuvo el 41,72\% de los sufragios y 8 de los 13 concejales. En ambos casos, hubo entre 8 y 6 candidaturas respectivamente, lo que fragmentó el voto y se vio beneficiada la fuerza más votada.

En algunos casos, esta diversidad política, si ha obtenido representación en los ayuntamientos, conformando un arco municipal multicolor con ediles de numerosas fuerzas políticas. Uno de los casos más significativos es la localidad de San Fernando de 
Henares, donde se reparten veintiún concejales entre nueve formaciones políticas. También son representativos, por el tamaño del municipio, los casos de Nuevo Baztán y El Boalo, con trece concejales cada uno, y con una amplia diversidad política con ediles de ocho y siete partidos diferentes respectivamente. Estos escenarios son cada vez más frecuentes en los consistorios, y ha sido necesaria una política de pactos y de coaliciones entre distintas fuerzas políticas, e incluso de diversa ideología política, para poder formar gobiernos y poner en marcha las administraciones locales. El dialogo y la capacidad de ceder ante algunas cuestiones, son requisitos imprescindibles en la nueva etapa política que se vive, no solo en los ayuntamientos de la CM, sino en el resto de ayuntamientos de España, en los gobiernos de las diputaciones provinciales y en los regionales.

Podemos decir que el sistema bipartidista que había instaurado en España, desde las Elecciones Generales de 1977 y hasta las de 2011, se ha interrumpido, al menos por el momento, como consecuencia de la aparición de nuevos partidos políticos que resultan claves para la gobernabilidad. Una situación que se ha repetido, con diversos partidos políticos, en otras regiones como Aragón, Andalucía, Islas Baleares o la Comunidad Valenciana, donde ha sido necesario el acuerdo de entre tres y cuatro fuerzas políticas.

A escala municipal, en la CM, y en las últimas elecciones de 2019, los partidos de Cs, VOX y el clúster de Otros (54,26\% de los votos), han superado en número de votantes al PP-PSOE juntos (45,74\% frente al 74,36\% que obtuvieron en las elecciones de 2011), unas cifras que demuestran el fin de la hegemonía bipartidista. Sin embargo, a pesar de estos datos, el bloque PP-PSOE ha conseguido un mayor número de concejales (61,06\%) frente a los obtenidos por las nuevas formaciones (38,94\%). La estructura de los nuevos partidos se encuentra menos desarrollada que la de las formaciones políticas tradicionales, y no presentan, por el momento, candidaturas en todo el espacio autonómico, en especial en las poblaciones de menos habitantes, lo que les repercute en la obtención de un mayor número de concejales.

El multipartidismo se ha asentado en el territorio madrileño, así como en el escenario político español, en todas las escalas, y todo parece indicar que, seguirá presente en los próximos años. Cabe preguntarnos ¿̇e mantendrá en el tiempo, o se volverá al sistema tradicional bipartidista como ha sucedido en otros países como Bolivia? o, sin embargo ¿esta será la nueva realidad política como sucede en países europeos como Alemania? ¿Cómo será la segmentación del voto en el territorio madrileño?, ¿Hacía que modelo político se dirige el territorio español?

\section{Agradecimientos}

Este trabajo se ha realizado en el marco del proyecto de investigación 03/2019 financiado por la Facultad de Geografía e Historia de la Universidad Nacional de Educación a Distancia (UNED) y que lleva por título "El efecto territorial de la evolución del voto en las elecciones municipales de la Comunidad de Madrid (2011-2019)". 


\section{Bibliografía}

Cabral, M.E. (1977). As eleicoes legislativas. Algunas perspectivas regionais. Lisboa: Livros Horizonte.

Archondo, R. (1999). Bolivia, el auge del multipartidismo. Nueva sociedad, 164, pp. 19-25.

Blanco, R.L. (2016). Fuerzas emergentes y fuerzas tradicionales en la democracia española. Revista de Libros. https://www.revistadelibros.com/articulo_imprimible.php?art=5240\&t=articulos

Blanco, R.L. (2017). El año que vivimos peligrosamente: del bipartidismo imperfecto a la perfecta ingobernabilidad. Revista Española de Derecho Constitucional, 109, pp. 63-93. https://doi.org/ 10.18042/cepc/redc.109.03

Bosque, J. (1982). Geografía electoral, Geografía política y Elecciones en España. Anales de Geografía de la Universidad Complutense, 2, pp. 263-274.

Cox, K.R. (1970). Geography, social contexts and voting behavior in Wales, 1861-1951. En Allardt, E. y Rokkan, S. (Coord.), Mass Politics. Nueva York: The Free Press.

De Montalvo, F. (2015). Multipartidismo territorial y forma de gobierno parlamentaria: la West Lothian Question como paradigma de los efectos del fenómeno. Revista española de derecho constitucional, 105, pp. 77-104. https://www.jstor.org/stable/24887422

Delgado, I. (1999). Resultados electorales y orientación del voto en los comicios municipales de 1995. Revista Española de Investigaciones Sociológicas, 86, pp. 247-273.

Delgado, I. (2010). Elecciones municipales en España. Dimensiones analíticas y aspectos distintivos de ocho procesos electorales (1997-2007). Politica y Sociedad, 47, pp. 13-36.

Gil-Torres, A. (2018). Bipartidismo y pluridemocracia en España: la construcción del contexto sociopolítico español según el CIS (2011-2016). index.comunicación, 8 (1), pp. 81-100.

Kaufmann, K. y Rodríguez, A. (2011). Political Behavior in the Context of Racial Diversity: The Case for Studying Local Politics. PS: Political Science and Politics, 44 (1), pp. 101-102. https:// doi.org/10.1017/S1049096510001952

Laux, H.D. y Simms, A. (1973). Parliamentary elections in West Germany: the geography of political choice. Area, 5, pp. 166-171.

Llera, J. (1998). Los rendimientos de los sistemas electorales de las comunidades autónomas: el predominio del bipartidismo imperfecto. Reis, 82, pp. 127-157.

Marschall, M., Shah, P. y Ruhil, A. (2011). The study of local elections. PS: Political Science \& Politics, 44(1), pp. 97-100. http://dx.doi.org/10.1017/s1049096510001940

Monzón, N.B. (2009). Geografía electoral. Consideraciones teóricas para el caso argentino. Cuadernos de Geografia, Revista Colombiana de Geografia, 18, pp. 119-128. https://doi.org/ 10.15446/rcdg.n18.13030

Riera, P., Gómez, R., Barberá, P., Mayoral, J.A. y Montero, J.R. (2016). Elecciones municipales en España: un análisis multinivel de los determinantes individuales y contextuales del voto. $R e-$ vista de Estudios Políticos, 172, pp. 47-82. 
Riera, P., Gómez, R., Mayoral, J.A., Barberá, P. y Montero, J.R. (2017). Elecciones municipales en España. La personalización del voto. Revista Internacional de Sociología, 75 (2), pp. 1-18. e062. http://dx.doi.org/10.3989/ris.2017.75.2.15.140

Sánchez, O. (2017). El fin (momentáneo) del bipartidismo en España: Análisis de los resultados electorales de 2015 y 2016. Revista Española de Derecho Constitucional, 109, pp. 237-260. https://doi.org/10.18042/cepc/redc.109.09

Scott, J. (1999). Multipartidismo, federalismo robusto y presidencialismo en Brasil. Araucaria: Revista Iberoamericana de filosofía, política y humanidades, 2, pp. 58-120.

Siegfried, A. (1913). Tableau politique de la France de l'Ouest sous la Troisième République. Bruselas: Editions de l'Université de Bruxelles.

Sodaro, M.J. (2006). Política y Ciencia Política. Una introducción. Madrid: Mcgraw-Hill.

Sonnleitner, W. (2017). Rastreando las dinámicas territoriales de la fragmentación partidista en México (1991-2015). América latina hoy: Revista de ciencias sociales, 75, pp. 23-54. https:// doi.org/10.14201/alh2017752354 
